Meta

Journal des tradlucteurs

Translators' Journal

\title{
De la coïncidence accidentelle à la coïncidence dirigée
}

La traduction comme savoir-faire et les modèles

logico-sémantiques qui en rendent compte

\section{Jeanne Dancette}

Volume 39, numéro 3, septembre 1994

URI : https://id.erudit.org/iderudit/002449ar

DOI : https://doi.org/10.7202/002449ar

Aller au sommaire du numéro

Éditeur(s)

Les Presses de l'Université de Montréal

ISSN

0026-0452 (imprimé)

1492-1421 (numérique)

Découvrir la revue

Citer cet article

Dancette, J. (1994). De la coïncidence accidentelle à la coïncidence dirigée : la traduction comme savoir-faire et les modèles logico-sémantiques qui en rendent compte. Meta, 39(3), 439-445. https://doi.org/10.7202/002449ar d'utilisation que vous pouvez consulter en ligne. 


\title{
DE LA COÏNCIDENCE ACCIDENTELLE À LA COIINCIDENCE DIRIGEEE - LA TRADUCTION COMME SAVOIR-FAIRE ET LES MODÉLES LOGICO-SÉMANTIQUES QUI EN RENDENT COMPTE
}

\author{
JEANNE DANCETTE \\ Université de Montréal, Montréal, Canado
}

«Même si le monde était un labyrinthe, nous ne pourrions le traverser sans respecter certains parcours obligés», dit Umberto Eco (1992: 9) dans son ouvrage au titre éloquent, Les limites de l'interprétation, dans lequel il dresse les limites de la théorie de la déconstruction du texte, permettant toutes les interprétations et donnant le beau rôle au lecteur prométhéen, toujours porteur d'un nouvelle lecture.

Le traducteur, comme le lecteur, suit une démarche généralement rationnelle et raisonnée; les hypothèses qu'il fait sur le sens du texte sont contrôlées et gardées dans les limites de ce qu'autorisent le texte et l'acte communicatif; les équivalences auxquelles il arrive sont rarement le fruit de hasards dus aux ressemblances formelles entre les langues, mais résultent bien plus souvent d'un travail d'analyse du sens du texte.

Le traducteur ne doit plus être vu comme la «victime de coïncidences accidentelles», mais comme le «générateur de cö̈ncidences intentionnelles», dit Beaugrande (1991: 30) dans un article où il invite à réviser les théories linguistiques qui ont privilégié la forme au détriment du sens, le système verbal au détriment du contexte communicatif. La traduction résulte du fait que le traducteur «can intentionally generate a coincidence by using resources and material to construct an event which is similar to a previous one despite unavoidable differences of time, place, and circumstance» (1991: 18). La coïncidence entre "actes de communication» est définie comme «a dialectic between similarity and difference». Elle peut être générée intentionnellement selon des principes dérivés non seulement des mots et des expressions ou des équivalents dictionnairiqués, mais de la textualité globale, poursuit l'auteur (1991: 26).

Misant sur les progrès de la linguistique du texte des sciences cognitives et sur l'apport de la traductologie aux sciences du langage, nous pensons qu'il doit être possible, à terme, de découvrir ces principes supérieurs expliquant la traduction et de contribuer à une théorie unificatrice de la traduction.

Cet article vise principalement à trouver une articulation entre trois objets distincts qui intéressent la traductologie:

a) les résultats du savoir-faire intuitif. - $\mathrm{La}$ traduction se pratique, avec un succès relatif, sans avoir à se connaître ;

b) les concepts traductologiques;

c) les modèles théoriques. - Ils expliqueraient le savoir-faire en dégageant les principes et les règles sur lesquels il repose et qui le rendent perfectible, reproductible et simulable (IA). 
Parmi tous les modèles pertinents pour la traduction, nous retiendrons les modèles logico-sémantiques fondés sur l'analyse propositionnelle et les relations prédicatives (Kintsch et van Dijk 1983; Mel'čuk 1978, 1981), car ils forment la base minimale de l'appréhension du sens en contexte et de traitement. Nous nous appuierons en outre sur les approches expérimentales (enregistrement des données verbales, entre autres), car ces approches créent les conditions scientifiques de la vérification du caractère opératoire des concepts et des thèses intuitives émises sur le savoir-faire.

Dans une première partie, nous décrirons des situations de traduction à la lumière des principes stratégiques et rationnels guidant la prise de décision, pour discuter dans une deuxième partie un modèle descriptif du processus de traduction qui tentera d'expliquer l'ancrage des principes traductologiques dans la pratique, la dynamique même de la traduction.

\section{DES SITUATIONS DE TRADUCTION — PRINCIPES STRATÉGIQUES ET RATIONNELS GUIDANT LA PRISE DE DÉCISION}

\section{Une démarche hésitante...}

Soit la phrase, extraite d'un rapport sur le développement portuaire de Vancouver, donnée dans son contexte à un groupe de traducteurs étudiants et professionnels 2 :

Vancouver is the spout of a single funnel receiving over $90 \%$ of all rail goods shipped overseas from Western Canada.

La difficulté rencontrée par tous les répondants portait sur le segment spout of a single funnel et se manifestait par un va-et-vient entre le transcodage et la traduction libre, explicative.

Nos observations ont montré que le processus de traduction intervient au niveau macroconceptuel et au niveau microconceptuel. La démarche macroconceptuelle, s'appuyant sur le reste du texte (ici, surtout la fin de la phrase), aboutissait à une périphrase explicative (au moins minimalement) telle que Vancouver est le lieu géographique/le point/le point d'arrivée. La démarche microconceptuelle s'attachait aux mots ou expressions isolés : spout, funnel, et à leurs équivalents dictionnairiques bec, entonnoir. Elle se soldait (au moins temporairement) par une traduction mot à mot: Vancouver est le bec d' un entonnoir unique (transcodage).

Cette deuxième opération n'exclut pas, toutefois, qu'il y ait un jugement sur la valeur de cette équivalence mot à mot, pour vérifier que celle-ci ne contredit pas l'interprétation macroconceptuelle; de même que la première opération n'exclut pas la possibilité pour le traducteur de retenir, pour quelque raison que ce soit, une traduction transcodée bec de l'entonnoir.

Nous émettons l'hypothèse, qui serait à vérifier sur un plus grand nombre de sujets, que la formulation de phrases explicatives (même incomplètes) constitue une étape nécessaire précédant le choix traductionnel, et que le travail de recherche d'équivalents passe par des productions de séries syntagmatiques (telles que les périphrases explicatives) et de séries paradigmatiques. Dans ce travail heuristique et raisonné se situe la coïncidence intentionnelle, dirigée.

Des principes stratégiques et rationnels peu contraignants...

1) Principe de la cohérence. - Dans l'exemple précédent, l'expression littérale bec de l'entonnoir n'a pas de cohérence au sens propre avec les concepts 'port', 'transport maritime' ou 'transport ferroviaire'. Le sujet doit alors faire l'hypothèse qu'il s'agit d'une métaphore et il en vérifie la cohérence avec le reste de la phrase: 'lieu où arrivent plus de $90 \%$ de tous les biens [...] 'point de convergence où aboutissent' [...]. Une telle décision sur l'interprétation est faite à l'issue de ce que Daniel Gile (1992) appelle «test de 
plausibilité». Notons ici que, face à une formulation qui peut paraître obscure (métaphore inusitée), l'interprétation est orientée par ce qui est connu. C'est ce noyau qui élucide le sens de la métaphore. Le graphe de Venn qui suit représente l'orientation de la démarche interprétative.

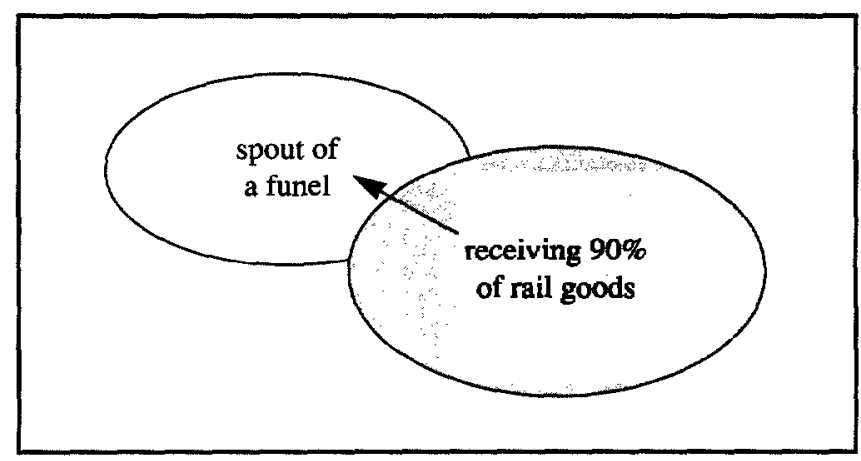

2) Principe de l'acceptabilité. - Il concerne la forme grammaticale, linguistique, rhétorique (ou autre) d'une équivalence. À l'issue d'un «test d'acceptabilité» (Gile), des décisions peuvent être prises pour marquer la préférence d'une forme sur une autre, par exemple point de convergence sur bec de l'entonnoir.

L'analyse des productions verbales des répondants indique que ces principes sont connus et évoqués mais que les choix traductionnels n'en sont pas une application stricte.

\section{Des principes contradictoires...}

Prenons un deuxième exemple, tiré de la littérature malgré les apparences.

$\mathrm{He}$ [Dick] managed to reach them [his children] over the head of employees on the principle that both the forcing of children and the fear of forcing them were inadequate substitutes for the long, careful watchfulness, the checking and balancing and reckoning of accounts, to the end that there should be no slip beyond a certain level of duty. (F. S. Fitzgerald, Tender is the Night)

Les opérations à l'œuvre dans le traitement de cette phrase sont orientées par des principes apparemment contradictoires: la nécessité de lever les ambiguïtés et la nécessité de les maintenir.

3) Désambiguïser. - Par quel processus? La recherche des relations prédicatives à l'intérieur des propositions semble élémentaire. Prenons, par exemple, le prédicat forcing. Il a pour actant objet children; mais quel est son actant sujet: Dick, employees ou adults in general? Le prédicat achieving dans «achieving a certain level of duty» est encore plus problématique. Qui a un devoir et envers qui? Ni l'actant sujet ni l'actant destinataire ne sont précisés.

Les deux réseaux qui suivent exploitent l'idée qu'un texte est une séquence de propositions connectées, porteuses d'information. L'auteur du Tractatus, Wittgenstein, propose une analyse du langage qui soit entièrement désambiguïsée 3 . Dans nos graphes, les propositions sont exprimées au moyen de prédicats et d'arguments. On rappellera que le PREDICAT est l'unité discursive qui indique le procès, qu'il soit exprimé dans la structure de surface du texte par un verbe, un nom, un adjectif, un adverbe ou même par une conjonction telle que ET, OU. (Ici les prédicats sont managed, reach, over, on, principle, forcing, fear, etc.) L'ARGUMENT (ou ACTANT) est l'unité qui exprime l'être ou l'objet qui 
participe au procès. Dans ces deux graphes, l'indice 1 des flèches correspond à l'actant sujet et l'indice 2, à l'actant objet.

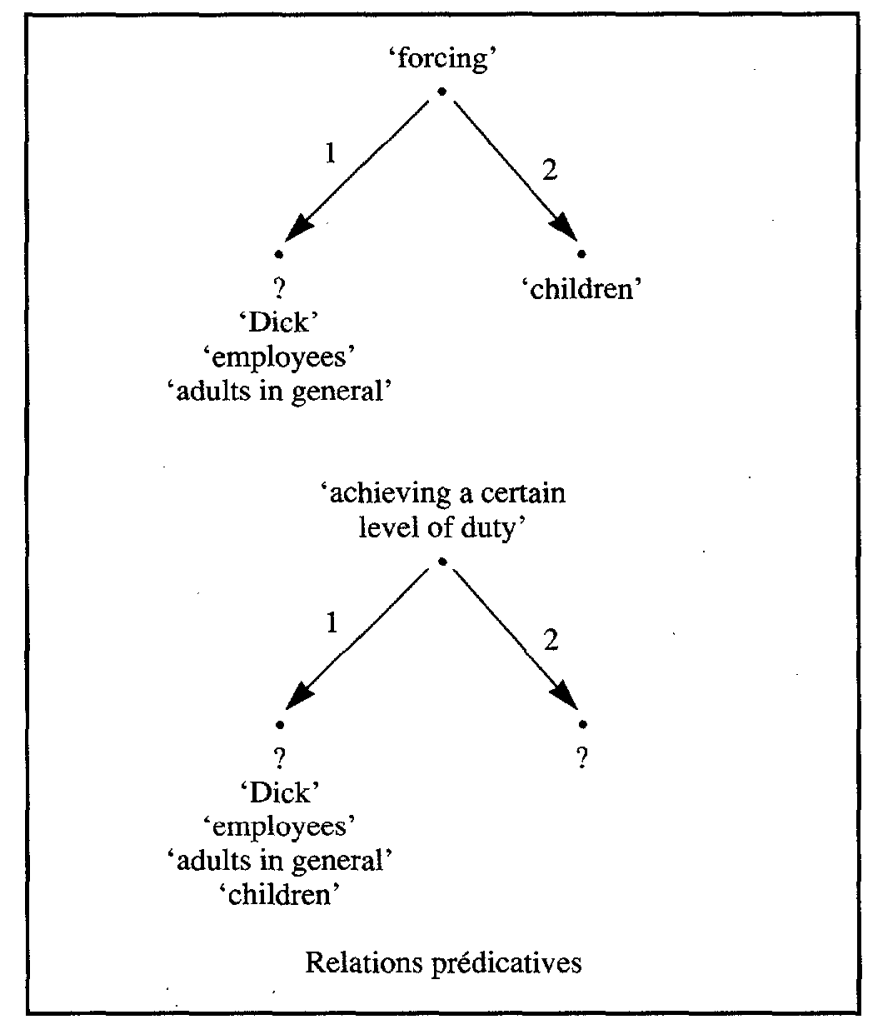

Ainsi le réseau (représenté ici sous une forme partielle, la plus élémentaire qui soit) est-il une formalisation du sens exprimé. Il présuppose, bien entendu, la description lexicale de chacune des étiquettes (unités sémantiques), mais faute d'espace, nous ne pouvons traiter de cet aspect qui relève de la sémantique du mot et de la lexicologie:

Le réseau illustre la démarche de désambiguïsation par la définition des relations prédicatives, au risque, toutefois, de tomber dans le piège de la traduction «hypertextuelle», rationalisante, clarifiante, allongeante, qui transforme polysémie voulue en monosémie (Berman 1985: 54).

4) Maintenir l'ambiguïté. - En suivant ce principe, au contraire, on fera une traduction plus littérale, selon la définition de Berman, fidèle à la forme, sans qu'elle soit nécessairement mot à mot. Nous notons, toutefois, que cet objectif n'est pas facile à atteindre. Quand elle n'est pas «hypertextuelle», la traduction littérale implique peut-être un soin encore plus grand dans les choix lexicaux pour laisser ouvertes toutes les options d'interprétation. En effet, pour maintenir l'ambiguïté, il faut déjà avoir fait plusieurs des lectures qu'autorise le texte original. 


\section{Un principe souvent négligé, le respect du droit de l'œuvre}

5) Aux principes précédents de cohérence et de plausibilité s'ajoute celui du «respect du droit de l'œuvre», de l'«interprétation selon l'intention du texte (intentio operis)». Ce principe revient à n'en dire ni plus ni moins que l'œuvre, à respecter le style, la concision, la forme, etc. «Les limites de l'interprétation s'arrêtent là où commencent les droits de l'œuvre.» (Eco 1992: 18).

La traduction du passage de Tender is the Night, faite par $\mathrm{J}$. Tournier, peut être évaluée à la lumière des quelques principes que nous avons dégagés. Les écarts ou désambiguiisations sont indiqués par les caractères gras.

Ignorant volontairement la présence des gouvernantes, Dick multipliait les contacts avec eux, partant du principe qu'en matière d'éducation trop demander aux enfants ou avoir peur de trop leur demander n'étaient pas des critères suffisants, et que la patience attentive, le contrôle suivi, l'équilibre et l'appréciation des résultats obtenus permettaient seuls de les maintenir à un niveau décent de conscience morale. (Fitzgerald 1985:316)

Laissons le lecteur libre d'apprécier cette traduction, admirable à bien des égards.

Contrairement aux conceptions linguistiques traditionnelles de la traduction, qui, selon Beaugrande, ont tendance à minimiser la question du sens (Bloomfield 1933: 93, $161,167,266,268$; Chomsky dans ses premiers modèles, 1957, etc.), nous avançons l'idée que la traduction est surtout un travail sur le sens. Des principes méthodologiques ci-dessus énoncés, le plus important serait alors celui de cohérence. La démarche (quelle que soit la forme finale de la traduction) doit passer par l'analyse du contenu propositionnel (prédicat et agents), par une certaine désambiguïsation et par la recherche de la cohérence du texte et de ses segments.

Nous voulons illustrer l'importance de l'extraction des propositions (au sens logicosémantique du terme) au moyen d'un autre exemple. Soit la phrase extraite d'un article de journal canadien :

In a dramatic reversal of predictions on Monday of a 40-basis point bank rate increase the key rate actually dropped one basis point to $8.94 \%$ at its weekly setting yesterday.

Cette phrase se caractérise par une très grande concision. Le contenu informatif doit en être dégagé pour que le transfert linguistique ait les meilleures chances de succès.

Série de propositions:

$\mathrm{P}_{\mathbf{a}} \quad=$ 'Les prédictions ont été complètement démenties'

$\mathrm{P}_{\mathrm{b}}^{\mathrm{a}} \quad=$ 'Les prédictions faites lundi étaient qu'il y aurait une augmentation de 40 points de base du taux d'escompte'

$\mathrm{P}_{\mathrm{c}} \quad=$ 'Le taux directeur (= taux d'escompte) a baissé de 1 point de base'

$P_{d}^{c} \quad=$ 'Il s'est fixé hier à $8,94 \%$ lors de l'adjudication hebdomadaire'

Rel $P_{a} \cdot b=P_{b}$ développe $P_{a}$

Rel $P_{a . c}^{a . b}=P_{c}^{b}$ explique $P_{a}$

$\operatorname{Rel} \mathrm{P}_{\text {a.d }}^{\text {a.c }}=\mathrm{P}_{\mathrm{d}}^{\mathrm{c}}$ explique $\mathrm{P}_{\mathrm{a}}^{\mathrm{a}}$.

Sur la base d'une analyse propositionnelle, nous rejetterons la traduction mot à mot suivante:

Lundi, dans un renversement dramatique des prévisions de 40 points de base du taux d'escompte, le taux directeur est tombé de 1 point de base à $8,94 \%$ à sa fixation hebdomadaire d'hier

car les relations temporelles et syntaxico-sémantiques qu'elle exprime ne sont pas conformes aux propositions dégagées ci-dessus. 
Ces quelques exemples avaient pour objet d'illustrer la place des raisonnements et des principes méthodologiques qui les sous-tendent. Si la démarche traductionnelle est nécessairement tâtonnante, elle n'en est pas pour autant arbitraire. La décrire, dégager les stratégies en cuvre relève de la méthode scientifique. Un schéma qui permettrait de retracer certaines des données prises en compte par le sujet traducteur dans son double travail de compréhension et de recherche des équivalences serait un progrès dans cette voie.

\section{SCHÉMA DE LA DOUBLE HÉLICE}

Nous proposons dans ce but un schéma qui vise à décrire certaines des opérations de base de la compréhension en traduction. En prenant la métaphore de la double hélice, le mouvement de la compréhension suit une courbe hélicoïdale ayant un point de départ et un point d'arrivée. La recherche des équivalences traductionnelles suit, elle aussi, une courbe hélicoïdale. Les deux courbes ne se confondent pas mais sont en rapport étroit. La double hélice s'arrête au point où un degré jugé satisfaisant de compréhension et d'équivalence interlinguistique est atteint.

Le fragment de la double hélice qui suit illustre le traitement possible de spout of a single funnel, vu dans notre premier exemple ${ }^{4}$.

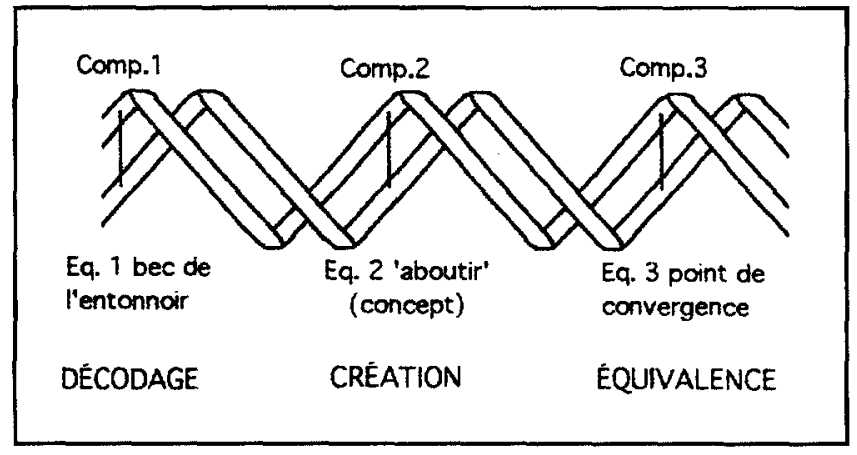

Au point de compréhension 1 (comp.1), au stade du décodage, le répondant trouve bec de l'entonnoir. Il n'est pas satisfait et lit la suite de la phrase. Il passe à un stade supérieur de compréhension (comp.2) au moment où il arrive à formuler une phrase-concept (stade de l'auto-explication ou création, Eq.2), et arrive enfin à une équivalence qui le satisfait : point de convergence.

Le schéma rend compte du passage entre les opérations linguistiques (décodage, analyse, transfert) et les opérations cognitives (émission, vérification, infirmation des hypothèses émises sur le sens, et même jugements métalinguistiques sur les énoncés). Il nous permet ainsi d'observer les rapports subtils entre unités linguistiques (dans chacune des deux langues) et concepts lors du passage du texte source au texte cible, entre démarche sémasiologique et démarche onomasiologique.

Cette formalisation illustre un processus intégré qui se joue à différents niveaux de structures linguistiques et en synchronie. Si un à un tous les éléments de la résolution des problèmes sont connus (perception de l'ambiguïté, élucidation du sens d'un mot par le contexte, recherche de la cohérence, etc.), par contre, c'est le dynamisme même du processus qu'il s'agit de décrire. Une validation du modèle en temps réel peut amener à introduire des contraintes telles que le saut de certaines opérations, des raccourcis, des reprises, dans des circonstances précises. 


\section{CONCLUSION}

Nous cherchons à analyser et à expliquer, dans un même schéma intégrateur, plusieurs problèmes de traduction. La réflexion qui préside à la traduction peut être suivie et, dans une certaine mesure, contrôlée par le sujet traduisant qui peut ainsi éviter les déviations et les erreurs de son raisonnement. Mieux, cette réflexion peut être guidée par des principes acquis, forgés dans la pratique et dans l'apprentissage.

Suivant des «parcours obligés», la réflexion du lecteur-traducteur s'exerce à trouver les coïncidences entre l'acte d'énonciation de l'auteur et le sien. En pédagogie de la traduction, on peut en tirer profit pour fournir à l'étudiant les outils permettant de nourrir et de guider cette réflexion et lui proposer les exercices l'entraînant à cette réflexion. Les exercices de paraphrasage, de résumé et de réécritures à partir de l'original sont des exercices appropriés.

Ainsi la méthode traductologique est-elle à la fois déductive - en application de principes et de règles - et inductive - par la voie de questions entraînant la prise de conscience du processus et la verbalisation des difficultés rencontrées.

\section{Notes}

1. Nous remercions le CRSH et le CAFIR de l'Université de Montréal dont l'apport financier a rendu possible cette recherche.

2. L'expérience mentionnée dans cet article est décrite dans Dancette (1992b).

3. Pour Wittgenstein (1961: 92), la proposition est la base du discours : $«$ Le sens de la proposition est son accord et son désaccord avec les possibilités de l'existence et de la non-existence des états de choses.»

4. Pour une description plus théorique du schéma, voir Dancette (1992b).

\section{RÉFÉRENCES}

BEAUGRANDE, R. de (1991a) : «Coincidence in Translation: Glory and Misery Again», Target, vol. 3, $\mathrm{n}^{\circ} 1$.

BEAUGRANDE, R. de (1991b) : Linguistic Theory: The Discourse of Fundamental Works, Londres, New York, Longman.

BEAUGRANDE, R. de et W. DRESSLER (1981): Introduction to Textlinguistics, Londres, Longman.

BERMAN, A. (1985): Les tours de Babel, Trans Europ Repress, Mauvezin.

BLOOMFIELD, L. (1933) : Language, Chicago, University of Chicago Press.

CHOMSKY, N. (1965) : Aspects of the Theory of Syntax, Cambridge, MIT.

DANCETTE, J. (1989): «La faute de sens en traduction, un essai de formalisation», TTR - Traduction, Terminologie et Rédaction, vol. $2, \mathrm{n}^{\circ} 2$.

DANCETTE, J. (1992a): «Modèles sémantiques et propositionnels de l'analyse de la fidélité en traduction», Meta, vol. 37, $\mathbf{n}^{\circ}$ 3, Montréal, Presses de l'Université de Montréal.

DANCETTE, J. (1992b) : «Des processus de traduction concomitants : compréhension et recherche d'équivalences», $A C L A$, vol. $14, \mathrm{n}^{\circ} 1$.

DANCETTE, J. (1994): Parcours de traduction. Étude expérimentale du processus de compréhension, Lille, PUL.

ECO, U. (1992): Les limites de l'interprétation, Grasset, traduction de I Limiti dell'interpretazione, Milan, Gruppo Editoriale Fabbri, Bompiani, Sonzogno, Etas S.p.A.

FITZGERALD, F. S. (1960): Tender is the Night, New York, Scribner; Tendre est la nuit (1985), trad. de l'anglais par J. Tournier, Paris, Belfond.

GILE, D. (1992): «Les fautes de traduction: une analyse pédagogique», Meta, vol. 37, $\mathrm{n}^{\circ} 2$, Montréal, Presses de l'Université de Montréal.

HOUSE, J. et S. BLUM-KULKA (dir.) (1986) : Interlingual and Intercultural Communication, Tübingen, Gunter Narr Verlag.

KINTSCH, W. (1988): «The Role of Knowledge in Discourse Comprehension: A Construction-integration Model», Psychological Review, vol. 95, $\mathrm{n}^{\circ} 2$

KINTSCH, W. et T. A. VAN DIJK (1983) : Strategies of Discourse Comprehension, New York, Academic Press. SNELL-HORNBY, M. (1988) : Translation Studies, An Integrated Approach, Amsterdam, John Benjamins. WITTGENSTEIN, L. (1961): Tractatus logico-philosophique, traduction française, Paris, Gallimard. 